\title{
Introduction of hyssopus officinalis $I$. into in vitro culture to optimize the conditions for obtaining callus tissues and microclonal propagation as a promising metod of innovative agrobiotechnologies
}

\author{
Elena Maslova $^{1 *}$, Natalya Gulya $^{2}$, Tatyana Perelugina ${ }^{1}$, Valeria Semykina ${ }^{1}$, and Elena Kalashnikova ${ }^{3}$ \\ ${ }^{1}$ Belgorod State National Research University, Belgorod, Russia \\ ${ }^{2}$ Biotech company «Biocad», Moscow, Russia \\ ${ }^{3}$ Russian State Agrarian University - Moscow Timiryazev Agricultural Academy
}

\begin{abstract}
The sterilization process of plant explants of $H$. officinalis was optimized when introduced into an in vitro culture, the most effective sterilization modes, optimal sterilizing agents, their exposure time and concentration were selected. Callus tissues and mini-plants of $H$. officinalis were obtained in vitro and the most optimal nutrient media were determined both for microclonal propagation and for the induction of callus tissue H. officinalis, which can be further used for mass cultivation of cell and culture and obtaining safe bio-additives with active substances for livestock and crop production as a part of the development modern agrobiotechnologies.
\end{abstract}

\section{Introduction}

Modern agrobiotechnology is currently the main priority area of development of the Russian Federation. To increase the efficiency of crop production and obtain greater productivity and yield of the target product, innovative agricultural and biotechnological methods are used. So, for example, microclonal propagation is used for further mass propagation and cultivation of highquality healthy plants by the traditional method in field and greenhouse conditions. The same method of cell and tissue culture can be used to obtain callus tissue of plants and to cultivate their cellular plant biomass in laboratory conditions and bioreactors in industrial biotechnological enterprises to obtain the target product, containing valuable biologically active substances or components with antibacterial, antimicrobial, antitumor and immunomodulating properties. They can be used in animal husbandry as a biological supplement and in crop production as a biological product that reduces the use of chemical additives and drugs that increase the immunity of both plant and animal organisms. One of such new promising sources is the representative of the Lamiaceae family - Hyssopus officinalis L. is a representative of the genus Hyssopus.

H. officinalis is a perennial semi-shrub plant that grows under natural conditions in the southern regions of the Russian Federation, the Caucasus, the Black Sea region, the Crimea, Altai, Central Asia, Europe and North Africa [1-2].

H. officinalis is a honey plant, as well as a medicinal and a spicy aromatic plant. It is included in the pharmacopoeias of France, Sweden, Germany, Romania,

*Corresponding author: maslova@bsu.edu.ru and Portugal [3]. It contains essential oil, sesquiterpenes, tannins, oleanolic and ursolic acids, resins, mineral salts, organic acids, flavonoids [2, 4].

It is known that in the flowering phase, the aerial mass of $\mathrm{H}$ officinalis contains up to $1.5 \%$ of essential oil in terms of dry weight; flavonoids (apigenin, luteolin, quercetin), their glycosides and phenolic acids (chlorogenic, protocatechuic, ferulic lilac, phydroxybenzoic coffee, vanillin, p-coumaric, rosmarin and gentisin); Vitamin $\mathrm{C}$ and carotene, along with many other substances [5-7]. The essential oil of $\mathrm{H}$. officinalis, which is obtained in many countries of the world, is of great interest [8-9]. The chemical composition of $H$. officinalis essential oil was studied in various parts of the world, and according to the results of most studies, the main components are pinocamphone and isopinocamphone [5-7, 10-13]. The composition of the essential oil and the quantitative content of various compounds differ depending on soil-climatic and genetic factors [13]. Nevertheless, the main components characteristic of this plant are pinocamphone, isopinocamphone, $\beta$ pinene, sabinene, myrcene, $\beta$ fellandren, linalool, myrtenol, elemol and germacren-D. Thymol and methyl acetate chemotypes of $H$. officinalis also grow in Iran [14-15]. Hyssopus vegetable raw materials and essential oils are used in perfumes and cosmetics (aromatic components of perfumes, cosmetics, soaps), food (spice and fragrant seasoning for flavouring cold snacks, meat and fish dishes, sauces, alcoholic beverages) and the pharmaceutical industry. Hyssopus has excellent phytoncidal properties [16-17]. 
Of particular value are the leaves of the plant. Hyssopus officinalis has anti-inflammatory, antiseptic, antitussive effects. It stimulates digestion and relieves spasms of the smooth muscles of the gastrointestinal tract $[1,18]$. Hyssopus infusions are used medicinally for diseases of the upper respiratory tract, cough, bronchitis, bronchial asthma, pneumonia and pulmonary tuberculosis, angina pectoris, neurosis, rheumatism, polyarthria, for the treatment of bruises, wounds, skin diseases [1]. This species has an expectorant, antispasmodic, decongestant, tonic effect, and some of its species exhibit a strong antiviral effect, especially against the herpes virus [18].

In experiments, conducted by scientists, Hyssopus officinalis exhibits antibacterial, antimycotic and antiprotozoal activity [19], and also has fungicidal properties [20].

Overall, it is clear that this culture is increasingly attracting the attention of scientists, due to the accumulation of a large number of biologically active compounds in plant tissues, which has been used in the pharmacy and food industry, and can also be used in modern agrobiotechnologies. Therefore, the relevance of studying of $H$. officinalis in an in vitro culture is evident and it is one of the priority areas of modern agrobiotechnology. To date, data on the study of the influence of cultivation conditions on clonal micropropagation and on the callus and morphogenesis of $H$. officinalis are not numerous [21-24], and the optimization of the in vitro cultivation process and the detailed study of this plant for the purpose of agrobiotechnology remains an urgent task.

The aim of our study is to optimize the sterilization process and the conditions for obtaining callus tissues and microclonal propagation as a promising culture for use in modern agrobiotechnologies.

\section{Experimental}

For research, the plant material of intact $\mathrm{H}$. officinalis plants growing on the territory of the Botanical Garden of the National Research University "BelSU" was collected. To introduce into in vitro culture and to obtain seedlings and mini plants, plant seeds were used as plant explants.

Sterilization was carried out in a stepwise manner according to generally accepted methods [25], using various disinfectants.

As sterilizing solutions, we used: sodium hypochlorite in a concentration of $5-15 \%$ and $2.5-7.5 \%$; lysoformin $3000-3 \%, 5 \%$ and $10 \%$; biocide - $3 \%, 5 \%$ and $10 \%$; silver nitrate $-0.1 \%$ and $0.5 \%$; chloramine B at a concentration of $5 \%$ and $10 \%$. Sterilizing solutions were left to expose to plant explants for 10, 20 and 30 minutes. At first, the plant material, washed in a soap solution, was placed in $70 \%$ ethyl alcohol for 1 minute, and then in a sterilizing solution of a certain concentration and for a certain time and after that it was washed three times in sterile distilled water. Sterile seeds were placed on the hormone-free Murashige-Skoog nutrient medium [26].

Further cultivation was carried out on a modified agar Murashige-Skoog medium [26] with a different composition of phytohormones (Table 1).

Nutrient media were made according to generally accepted in vitro culture methods for plant cells and tissues (the hormone-free Murashige-Skoog medium was used as a control) [27].

All work and manipulations with cultures were carried out under aseptic conditions in the Lamsystems laminar box of protection class II, type A2, according to generally accepted sterility practices when working with cell and tissue cultures [27-28].

After seed germination the seedlings were transferred, under aseptic conditions, to nutrient media containing various concentrations of phytohormones and cultivated in a light room at a temperature of $23{ }^{\circ} \mathrm{C}$ with observance of the day / night regime: 16/8 hours for 30 days.

Table 1. Composition of modified Murashige-Skoog nutrient media

\begin{tabular}{|c|c|c|c|c|c|c|c|c|c|}
\hline \multirow{2}{*}{ Components of the medium } & $\mathbf{M S}_{\mathbf{1}}$ & $\mathbf{M S}_{\mathbf{2}}$ & $\mathbf{M S}_{\mathbf{3}}$ & $\mathbf{M S}_{\mathbf{4}}$ & $\mathbf{M S}_{\mathbf{5}}$ & $\mathbf{M S}_{\mathbf{6}}$ & $\mathbf{M S}_{\mathbf{7}}$ & $\mathbf{M S}_{\mathbf{8}}$ \\
\cline { 2 - 10 } & \multicolumn{7}{|c|}{ Volume per 1 L } \\
\hline Macrosalt stock solutions, ml & 100 & 100 & 100 & 100 & 100 & 100 & 100 & 100 \\
\hline Microsalt stock solutions, ml & 1 & 1 & 1 & 1 & 1 & 1 & 1 & 1 \\
\hline Fe-chelate, ml & 5 & 5 & 5 & 5 & 5 & 5 & 5 & 5 \\
\hline $\mathrm{CaCl}_{2}, \mathrm{ml}$ & 5 & 5 & 5 & 5 & 5 & 5 & 5 & 5 \\
\hline Vitamins: PP, mg & 0.5 & 0.5 & 0.5 & 0.5 & 0.5 & 0.5 & 0.5 & 0.5 \\
\hline B $_{1}$ & 0.1 & 0.1 & 0.1 & 0.1 & 0.1 & 0.1 & 0.1 & 0.1 \\
\hline B $_{6}$ & 0.5 & 0.5 & 0.5 & 0.5 & 0.5 & 0.5 & 0.5 & 0.5 \\
\hline Mesoinositis, mg & 100 & 100 & 100 & 100 & 100 & 100 & 100 & 100 \\
\hline Glycine, ml & 2 & 2 & 2 & 2 & 2 & 2 & 2 & 2 \\
\hline$\alpha$-Naphthaleneacetic acid (NAA), mg & - & - & - & - & - & 0.5 & 2 & - \\
\hline Indole-3-acetic acid (IAA), mg & - & - & 1 & - & 2 & - & 2 & 3 \\
\hline Indole-3-butyric acid (IBA), mg & - & - & - & 1 & - & - & - & - \\
\hline Kinetin, mg & - & - & - & - & 0.2 & 0.1 & 1 & 2 \\
\hline 6-benzylaminopurine (6-BAP), mg & 1 & 3 & - & - & - & 1 & 1 & - \\
\hline 2,4-Dichlorophenoxyacetic acid (2,4-D), mg & - & - & - & - & - & - & 2 & - \\
\hline Sucrose, g & 20 & 20 & 20 & 20 & 20 & 20 & 20 & 20 \\
\hline Agar-agar, g & 7 & 7 & 7 & 7 & 7 & 7 & 7 & 7 \\
\hline
\end{tabular}


The quality of seedling growth in various nutrient media was assessed at weeks 1,2 and 3 of cultivation using morphometric indicators such as shoot length in centimetres, average number of leaves in pieces, root length and the presence or absence of wilting and discoloration of leaves.

Statistical data was treated using methods of mathematical statistics with the calculation of the arithmetic average, its error, and the Fisher criterion in Microsoft Office Excel [29].

\section{Results and discussion}

As a result of experimental work in vitro, optimal sterilizing agents of $\mathrm{H}$. officinalis were determined and data was obtained on the effect of the most effective sterilizing agent and its sterilization mode. The effect of sterilizing agents on obtaining sterile and viable explants of $\mathrm{H}$. officinalis during seed sterilization, as well as sterilization modes are presented in Table 2 and Figure 1.

According to Fisher's exact criterion, at a significance level of $\mathrm{P}>0.05$, in terms of its effect on the number of sterile explants, all the obtained data presented in the table significantly differ from the control.

As it can be seen from table 2 and figure 1, the optimal sterilizing agents for $\mathrm{H}$. officinalis seeds are a 5$15 \%$ solution of sodium hypochlorite with its effect for 10 minutes and a $10 \%$ solution of chloramine $\mathrm{B}$ with its effect for 10 minutes, since under these conditions the maximum ratio of the number of sterile $(100 \%)$ explants to the number of viable explants $(100 \%)$ was obtained. It is also possible to sterilize seeds with $3 \%$ biocide for 30 minutes ( $86.0 \%$ viable explants), $0.1 \%$ silver nitrate with an exposure time of 10 minutes $(80.33 \%)$. Sterilization with $10 \%$ chloramine $\mathrm{B}$ for 20 minutes, $5 \%$ chloramine $\mathrm{B}$ for 20 and 30 minutes and 5-15\% sodium hypochlorite for 20 minutes gives the same yield of viable explants (60.67\%). You can also use 5\% lysoformin 3000 for 10 minutes (59.67\% viable explants) and $5 \%$ biocide over time exposure time of 20 minutes $(57.3 \%$ of viable explants). It is not advisable to use all other sterilization modes, since they produce less number of viable plant explants (less than 50\%) or have a detrimental effect on the seeds.

In the course of the work the selection of the optimal composition of nutrient media was carried out to obtain mini plants of $H$. officinalis L., and callus cultures in vitro.

Plant sprouts of $H$. officinalis were cultivated on different media (the compositions are described in the research methods). The obtained parameters of the growth and development of mini-plants are presented in table 3, where SL is the shoot length, NL is the number of leaves, DR is the darkening of the root, $\mathrm{CP}$ is the color of the plant.

Based on the obtained data, the optimal medium for obtaining mini plants of the $\mathrm{H}$. officinalis species is $\mathrm{MS}_{5}$ medium, where there are more leaves, a more developed root system, and, in general, a more viable state of plants compared to other media. Optimum media for the induction of callus genesis are $\mathrm{MS}_{4}$ and $\mathrm{MS}_{6}$.

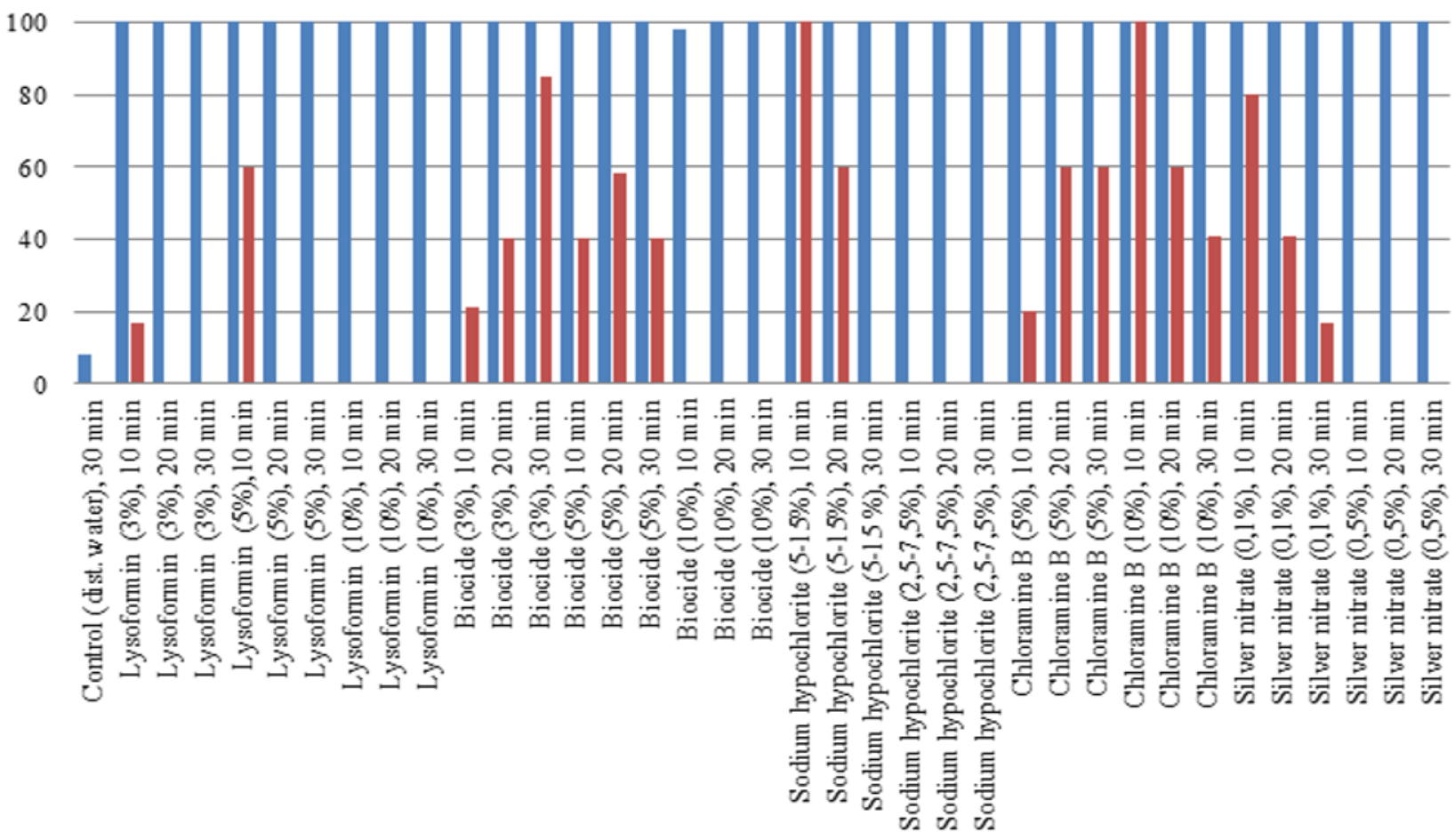

Num ber of sterile ex plants (\%) $\quad$ Num ber of viable ex plants (\%)

Fig 1. The effect of sterilizing agents on the number of sterile and viable explants of $H$. officinalis 
Table 2. The effect of sterilizing solutions on plant explants $H$. officinalis

\begin{tabular}{|c|c|c|c|}
\hline $\begin{array}{l}\text { Sterilizing solution and its } \\
\text { concentration }\end{array}$ & $\begin{array}{c}\text { Sterilization duration } \\
\text { (minutes) }\end{array}$ & Sterile explants number $(\%)$ & $\begin{array}{c}\text { Viable } \\
\text { explants number (\%) }\end{array}$ \\
\hline Lysoformin $3000(3 \%)$ & 10 & $99.67 \pm 0.11$ & $14.67 \pm 0.11$ \\
\hline Lysoformin 3000 (3\%) & 20 & $100.0 \pm 0.0$ & $0.0 \pm 0.0$ \\
\hline Lysoformin 3000 (3\%) & 30 & $100.0 \pm 0.0$ & $0.0 \pm 0.0$ \\
\hline Lysoformin $3000(5 \%)$ & 10 & $100.0 \pm 0.0$ & $59.67 \pm 0.11$ \\
\hline Lysoformin 3000 (5\%) & 20 & $100.0 \pm 0.0$ & $0.0 \pm 0.0$ \\
\hline Lysoformin $3000(5 \%)$ & 30 & $100.0 \pm 0.0$ & $0.0 \pm 0.0$ \\
\hline Lysoformin $3000(10 \%)$ & 10 & $100.0 \pm 0.0$ & $0.0 \pm 0.0$ \\
\hline Lysoformin $3000(10 \%)$ & 20 & $100.0 \pm 0.0$ & $0.0 \pm 0.0$ \\
\hline Lysoformin $3000(10 \%)$ & 30 & $100.0 \pm 0.0$ & $0.0 \pm 0.0$ \\
\hline Biocide $(3 \%)$ & 10 & $100.0 \pm 0.0$ & $21.0 \pm 0.2$ \\
\hline Biocide (3\%) & 20 & $100.0 \pm 0.0$ & $40.33 \pm 0.11$ \\
\hline Biocide (3\%) & 30 & $100.0 \pm 0.0$ & $86.0 \pm 0.2$ \\
\hline Biocide $(5 \%)$ & 10 & $100.0 \pm 0.0$ & $40.33 \pm 0.11$ \\
\hline Biocide $(5 \%)$ & 20 & $100.0 \pm 0.0$ & $57.3 \pm 2.3$ \\
\hline Biocide $(5 \%)$ & 30 & $100.0 \pm 0.0$ & $41.0 \pm 0.2$ \\
\hline Biocide (10\%) & 10 & $98.0 \pm 0.2$ & $0.0 \pm 0.0$ \\
\hline Biocide $(10 \%)$ & 20 & $100.0 \pm 0.0$ & $0.0 \pm 0.0$ \\
\hline Biocide $(10 \%)$ & 30 & $100.0 \pm 0.0$ & $0.0 \pm 0.0$ \\
\hline Sodium hypochlorite (5-15\%) & 10 & $100.0 \pm 0.0$ & $100.0 \pm 0.0$ \\
\hline Sodium hypochlorite (5-15\%) & 20 & $100.0 \pm 0.0$ & $60.67 \pm 0.21$ \\
\hline Sodium hypochlorite (5-15\%) & 30 & $100.0 \pm 0.0$ & $0.0 \pm 0.0$ \\
\hline Sodium hypochlorite $(2,5-7,5 \%)$ & 10 & $100.0 \pm 0.0$ & $0.0 \pm 0.0$ \\
\hline Sodium hypochlorite $(2,5-7,5 \%)$ & 20 & $100.0 \pm 0.0$ & $0.0 \pm 0.0$ \\
\hline Sodium hypochlorite $(2,5-7,5 \%)$ & 30 & $100.0 \pm 0.0$ & $0.0 \pm 0.0$ \\
\hline Chloramine B (5\%) & 10 & $100.0 \pm 0.0$ & $20.33 \pm 0.11$ \\
\hline Chloramine B (5\%) & 20 & $100.0 \pm 0.0$ & $60.67 \pm 0.21$ \\
\hline Chloramine B $(5 \%)$ & 30 & $100.0 \pm 0.0$ & $60.67 \pm 0.21$ \\
\hline Chloramine B (10\%) & 10 & $100.0 \pm 0.0$ & $100.0 \pm 0.0$ \\
\hline Chloramine B (10\%) & 20 & $100.0 \pm 0.0$ & $60.67 \pm 0.21$ \\
\hline Chloramine B $(10 \%)$ & 30 & $100.0 \pm 0.0$ & $41.0 \pm 0.2$ \\
\hline Silver nitrate $(0,1 \%)$ & 10 & $100.0 \pm 0.0$ & $80.33 \pm 0.11$ \\
\hline Silver nitrate $(0,1 \%)$ & 20 & $100.0 \pm 0.0$ & $41.0 \pm 0.2$ \\
\hline Silver nitrate $(0,1 \%)$ & 30 & $100.0 \pm 0.0$ & $14.67 \pm 0.11$ \\
\hline Silver nitrate $(0,5 \%)$ & 10 & $100.0 \pm 0.0$ & $0.0 \pm 0.0$ \\
\hline Silver nitrate $(0,5 \%)$ & 20 & $100.0 \pm 0.0$ & $0.0 \pm 0.0$ \\
\hline Silver nitrate $(0,5 \%)$ & 30 & $100.0 \pm 0.0$ & $0.0 \pm 0.0$ \\
\hline Control (distilled water) & 30 & $6.0 \pm 0.2$ & $0.0 \pm 0.0$ \\
\hline
\end{tabular}


Table 3. Cuitivation of $H$. officinalis on different types of culture media in invitro

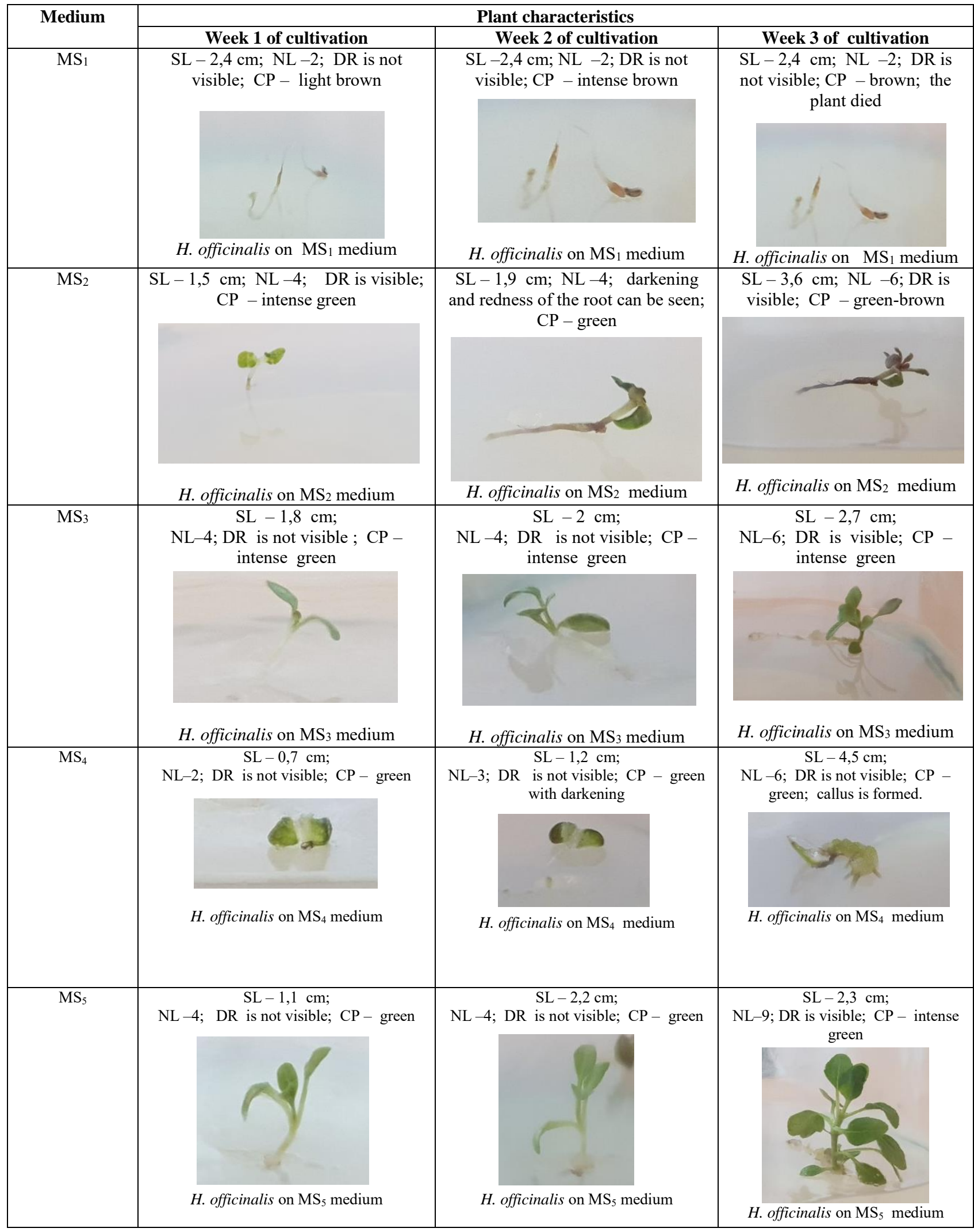




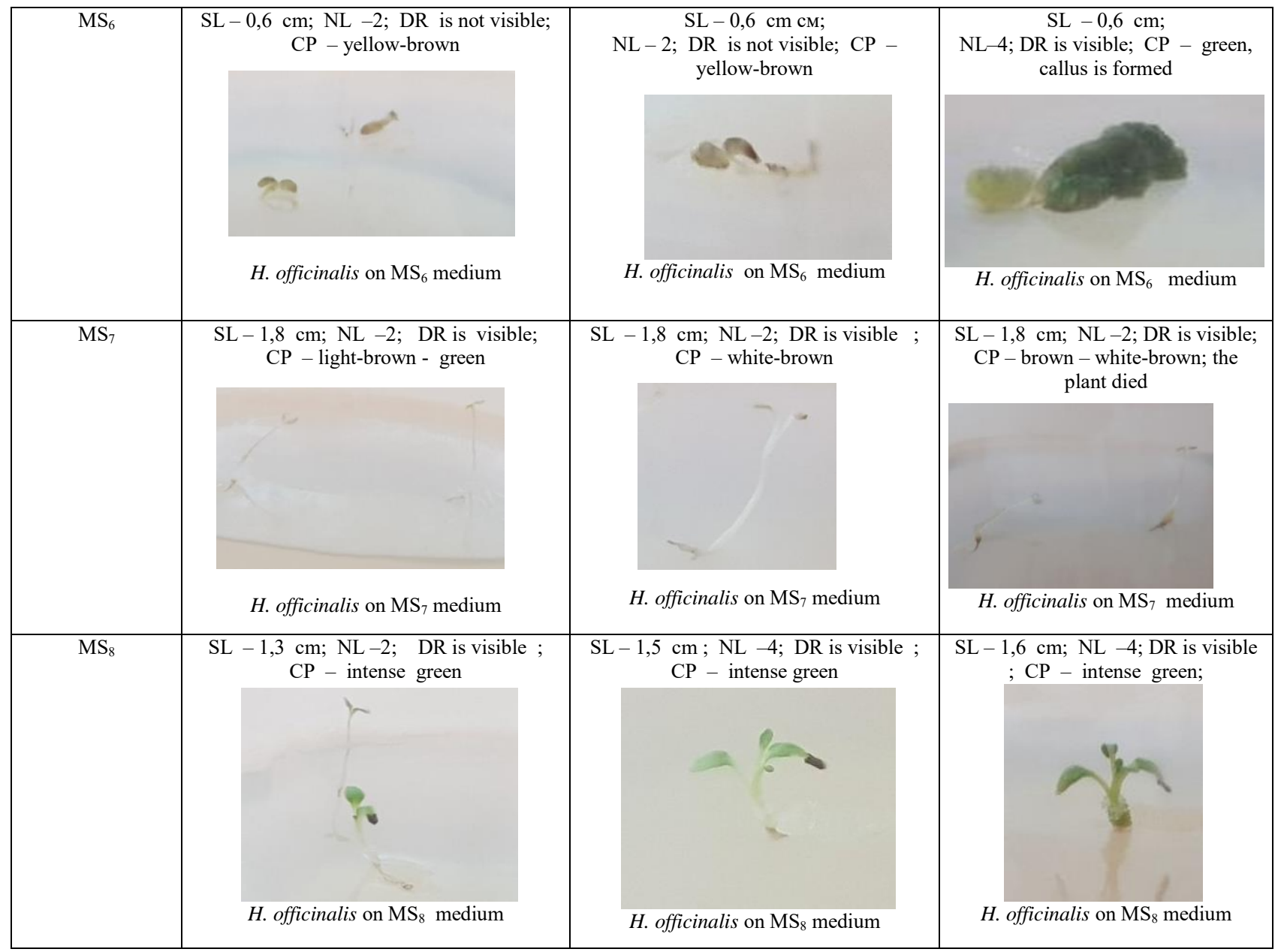

\section{Conclusion}

During the study, the introduction of $H$. officinalis plant explants into the in vitro culture was carried out, the sterilization process of $H$. officinalis plant explants was optimized, somel of the most effective seed sterilization modes, their exposure time and concentration were found. The most optimal are $5-15 \%$ solution of sodium hypochlorite and $10 \%$ solution of chloramine $\mathrm{B}$ with its effect for 10 minutes, providing $100 \%$ sterilization of plant explants and most importantly $100 \%$ germination of viable seedlings.

Eight culture media were tested for the cultivation of $H$. officinalis seedlings. It was found that the most optimal nutrient medium for the induction of callusogenesis was a medium with indolyl-3-butyric acid $-1 \mathrm{mg} / \mathrm{l}$, and a medium with a-naphthylacetic acid - 0.5 $\mathrm{mg} / 1$, kinetin $-0.1 \mathrm{mg} / 1$ and 6 -benzylaminopurine - 1 $\mathrm{mg} / 1$, and for the cultivation of plants $\mathrm{H}$. officinalis in vitro - a medium containing indolyl-3-acetic acid IAA (2 $\mathrm{mg} / \mathrm{l})$, and kinetin $(0.2 \mathrm{mg} / \mathrm{l})$.

Thus, callus tissues and mini-plants of $H$. officinalis were obtained in vitro and the most optimal nutrient media were determined both for microclonal propagation and for the induction of callus tissue $H$. officinalis, which can be further used for mass cultivation of cell and culture and production from it safe bioadditives with active substances for livestock and crop production as part of the development of modern agrobiotechnology.

\section{Acknowledge}

This work was carried out as part of a state task FZWG-2020-0021.

\section{References}

1 S. V. Sizonenko and T. A. Belova, "Hyssopus officinalis to the culture in Medicinal plant growing: from the experience of the past to the latest technologies: materials of the International scientific and practical Internet conference, Poltava, 2012.

2 V. E. Torikov, "Medicinal value of vegetable, fruit and berry, field plants and wild plants," (Publishing house of the Bryansk state agricultural Academy, Bryansk, 2013)

3 K. S. Timchuk, T. G. Zheleznyak, Z. N. Vorniku, and I. P. Dragalin, "Medicinal Hyssop - the content of essential oil and its component composition," Materials of the international scientific conference (may 29-31, 2013, Minsk)

4 O. A. Grebennikova, A. E. Paliy, L. A. Khlypenko, V. D. Rabotyagov, Journal "Orbital" 1, 1 (2017)

5 F. Fathiazad, S. Hamedeyazdan, Afr. J. Pharm. Pharmacol 5(17), 1959 (2011)

6 G. Zawiślak, Mod. Phytomorphol. 4, 93 (2013)

7 A. Srivastava, K. Awasthi, B. Kumar, A. Misra, S. Srivastava, India Pharmacogn J. 10(4), 690 (2018)

8 L. A. Kotyuk, Biologichni Studii 7(2), 83 (2013) 
9 A. N. Shibko and Yu. Aksenov, Ecosystems, their optimization and protection, 4, 127-133 (2011).

10 A. Wesołowska, D. Jadczak, M. Grzeszczuk, Herba Pol. 56(1), 57 (2010)

11 V. Mitić, S. Đorđević, FU Phys. Chem. Tech. 2(2), 105 (2000)

12 M. Moghtader, J. Hortic. For. 6(1), 1 (2014)

13 H. Soleimani, M. Barzegar, M. A. Sahari, B. H. Naghdi J. of Med. Plants, 10(37), 61 (2011)

14 G. Schulz, E. Stahl, Flavor Fragr. J. 6(1), 69 (1991)

15 N. Dehghanzadeh, S. Ketabchi, A. Alizadeh, Asian J. Exp. Biol. Sci. 3(4), 767 (2012)

16 M. Jankovský, T. Landa, Hort. Sci. (Prague), 29(3), 119 (2002)

17 F. Fathiazad, M. Mazandarani, S. Hamedeyazdan, Adv. Pharm. Bull. 1(2), 63 (2011)

18 M. A. Myadelets, D. V. Domrachev, V. A. Cheremushkina, Chemistry of plant raw materials $\mathbf{1}$, 111 (2012)

19 A. L. Budantsev and E. E. Lesiovskaya, Wild useful plants of Russia (SPKHFA Publishing house, Saint Petersburg, 2001)

20 D. Tančinová, Z. Mašková, D. Foltinová, J. Štefániková, J. Árvay, Potr. S. J. F. Sci. 12(1), 491 (2018)
21 T. G. Kuchina, I. K. Lebedev, and E. A. Kalashnikova, Study of medicinal hyssop (Hyssopus officinalis L.) in culture in vitro modern technologies in the world scientific space collection of articles of the International scientific and practical conference (September 28, 2017, Ufa).

22 B. Hosseini, M. Alizadeh, A. Hassani, M. Jafari, A. Rahimi, J. Medicinal Plants By-products 2, 187 (2016)

23 E. Zayova, M. Geneva, I. Stancheva, L. Dimitrova, M. Petrova, M. Hristozkova, I. Salamon, Med. Plants, 10(4), 295 (2018)

24 I. Stancheva, M. Geneva, M. Hristozkova, E. Zayova, J. Herbs Spices Med. Plants 25(2), 104 2019

25 A.V. Kilchevsky, Genetic bases of plant breeding: Biotechnology in plant breeding. Cell Engineering. (Nauka, Minsk, 2012).

26 T. Murashige, Physiol Plant 15(3), 473 (1962)

27 V. S. Shevelukha, Agricultural Biotechnology. (Vyshaya Shkola, Moscow, 2008)

28 O. A. Timofeeva and N. I Rumyantseva, Culture of plant cells and tissues (Kazan University, Kazan, 2012)

29 Pifo Hans-Peter. Statistics.( Ed. VNIIA, Moscow, 2011) 